\title{
Chemical differentiation in a prestellar core traces non-uniform illumination $\star, \star \star$
}

\author{
S. Spezzano ${ }^{1}$, L. Bizzocchi ${ }^{1}$, P. Caselli ${ }^{1}$, J. Harju ${ }^{1,2}$, and S. Brünken ${ }^{3}$ \\ 1 Max Planck Institute for Extraterrestrial Physics, Giessenbachstrasse 1, 85748 Garching, Germany \\ e-mail: spezzano@mpe.mpg.de \\ 2 Department of Physics, PO Box 64, University of Helsinki, 00100 Helsinki, Finland \\ 3 I. Physikalisches Institut, Universität zu Köln, Zülpicher Str. 77, 50937 Köln, Germany
}

Received 6 April 2016 / Accepted 7 July 2016

\begin{abstract}
Dense cloud cores present chemical differentiation because $\mathrm{C}$ - and $\mathrm{N}$-bearing molecules are distributed differently, the latter being less affected by freeze-out onto dust grains. In this letter we show that two C-bearing molecules, $\mathrm{CH}_{3} \mathrm{OH}$ and $c$ - $\mathrm{C}_{3} \mathrm{H}_{2}$, present a strikingly different (complementary) morphology while showing the same kinematics towards the prestellar core L1544. After comparing their distribution with the large-scale $\mathrm{H}_{2}$ column density $N\left(\mathrm{H}_{2}\right)$ map from the Herschel satellite, we find that these two molecules trace different environmental conditions in the surrounding of L1544: the $c-\mathrm{C}_{3} \mathrm{H}_{2}$ distribution peaks close to the southern part of the core, where the surrounding molecular cloud has an $N\left(\mathrm{H}_{2}\right)$ sharp edge, while $\mathrm{CH}_{3} \mathrm{OH}$ mainly traces the northern part of the core, where $N\left(\mathrm{H}_{2}\right)$ presents a shallower tail. We conclude that this is evidence of chemical differentiation driven by different amounts of illumination from the interstellar radiation field: in the south, photochemistry maintains more $\mathrm{C}$ atoms in the gas phase, allowing carbon-chain (such as $c-\mathrm{C}_{3} \mathrm{H}_{2}$ ) production; in the north, $\mathrm{C}$ is mainly locked in $\mathrm{CO}$, and methanol traces the zone where $\mathrm{CO}$ starts to freeze out significantly. During the process of cloud contraction, different gas and ice compositions are thus expected to mix towards the central regions of the core, where a potential solar-type system will form. An alternative view on carbon-chain chemistry in star-forming regions is also provided.
\end{abstract}

Key words. ISM: molecules - ISM: individual objects: L1544 - radio lines: ISM - ISM: clouds

\section{Introduction}

Prestellar cores represent the initial conditions of star formation, and they are highly valuable laboratories for studying physical and chemical processes without the complications that are due to protostellar feedback. Their chemical composition provides the starting point out of which the future protoplanetary disk and stellar system will form. Molecular studies also provide important clues on the dynamical status and evolution. Based on spectroscopic data, Keto et al. (2015) concluded that the prestellar core L1544 is slowly contracting and its kinematics is not consistent with the singular isothermal sphere (Shu 1977) and Larson-Penston (Larson 1969; Penston 1969) contraction. Caselli et al. (1999, 2002) showed that CO is heavily (>99\%) frozen onto the surface of dust grains in the central $6500 \mathrm{AU}$ of L1544, while $\mathrm{N}_{2} \mathrm{H}^{+}$and in particular $\mathrm{N}_{2} \mathrm{D}^{+}$better follow the millimetre dust continuum emission showing no clear signs of depletion (see also Crapsi et al. 2007, for similar conclusions on $\left.\mathrm{NH}_{2} \mathrm{D}\right)$. Tafalla et al. $(2002,2004,2006)$ extended this study to more starless cores and more molecular species. They found a systematic molecular differentiation, pointing out that $\mathrm{C}$-bearing species, such as $\mathrm{CO}, \mathrm{CS}, \mathrm{C}_{2} \mathrm{~S}, \mathrm{CH}_{3} \mathrm{OH}, \mathrm{C}_{3} \mathrm{H}_{2}$, and $\mathrm{HC}_{3} \mathrm{~N}$ are severely affected by freeze-out, showing a sharp central hole

\footnotetext{
^ Based on observations carried out with the IRAM $30 \mathrm{~m}$ Telescope. IRAM is supported by INSU/CNRS (France), MPG (Germany), and IGN (Spain).

$\star \star$ The reduced data are only available at the CDS via anonymous ftp to cdsarc.u-strasbg. fr (130.79.128.5) or via

http://cdsarc.u-strasbg.fr/viz-bin/qcat?J/A+A/592/L11
}

(with some differences in hole size depending on the particular molecule), while $\mathrm{N}_{2} \mathrm{H}^{+}$and $\mathrm{NH}_{3}$ seem present in the gas phase at the core centres. Carbon-chain molecules are known to preferentially trace starless and less evolved cores (Suzuki et al. 1992), where $\mathrm{C}$ atoms have not yet been mainly locked in CO. CCS has also been found in the outer outer edge of L1544 (Ohashi et al. 1999) and, in the case of L1551, with a clear shift in velocity compared to the LSR core velocity (Swift et al. 2006), indicating possible accretion of material from the more diffuse molecular cloud where the dense core is embedded. Although gas-grain chemical models (of spherically symmetric starless cores) can explain the observed differential distribution of $\mathrm{C}$ and N-bearing molecules based on the chemical/dynamical evolution (e.g. Aikawa et al. 2001), observational and theoretical studies have so far not discussed possible differences in the distribution of C-bearing molecules that are affected by central freeze-out. Here we report on the distribution of methanol $\left(\mathrm{CH}_{3} \mathrm{OH}\right)$ and cyclopropenylidene $\left(c-\mathrm{C}_{3} \mathrm{H}_{2}\right)$ towards $\mathrm{L} 1544$ and show that their complementary distribution can be understood if the non-homogeneous environmental conditions are taken into account.

The recent $\mathrm{CH}_{3} \mathrm{OH}$ map of Bizzocchi et al. (2014) shows an asymmetric structure, consistent with central depletion and preferential release in the region where $\mathrm{CO}$ starts to significantly freeze out $\left(\mathrm{CH}_{3} \mathrm{OH}\right.$ is produced on the surface of dust grains by successive hydrogenation of $\mathrm{CO}$; e.g. Watanabe \& Kouchi 2002). In particular, the $\mathrm{CH}_{3} \mathrm{OH}$ column density map presents a clear peak towards the north of the millimetre dust continuum 
peak (see Fig. 1 of Bizzocchi et al. 2014), thus revealing a nonuniform distribution around the ring-like structure. With the aim of exploring chemical processes across L1544, we mapped the emission of another carbon-bearing molecule, $c-\mathrm{C}_{3} \mathrm{H}_{2}$, which is representative of carbon-chain chemistry (Sakai et al. 2008). In this letter we investigate the effects of physical parameters on the distribution of methanol and cyclopropenylidene in L1544, and the influence of environmental effects on the gas- and grainphase chemistry.

\section{Observations}

\subsection{Cyclopropenylidene}

The emission map of cyclopropenylidene towards L1544 has been obtained in October 2013 using the IRAM 30 m telescope (Pico Veleta, Spain), during the same observing run in which methanol was observed (Bizzocchi et al. 2014). We performed a $2.5^{\prime} \times 2.5^{\prime}$ on-the-fly (OTF) map centred on the source dust emission peak $\left(\alpha_{2000}=05^{\mathrm{h}} 04^{\mathrm{m}} 17 \mathrm{~s} 21, \delta_{2000}=+25^{\circ} 10^{\prime} 42^{\prime \prime}: 8\right)$. The reference position was set at $\left(-180^{\prime \prime}, 180^{\prime \prime}\right)$ offset with respect to the map centre. The observed transitions are summarised in Table 1. The $\mathrm{CH}_{3} \mathrm{OH}$ observations are described in Bizzocchi et al. (2014). An additional setup was used to observe one line of $c-\mathrm{C}_{3} \mathrm{H}_{2}$. The EMIR E090 receiver was used with the Fourier Transform Spectrometer backend (FTS) with a spectral resolution of $50 \mathrm{kHz}$. The antenna moved along an orthogonal pattern of linear paths separated by $8^{\prime \prime}$ intervals, corresponding to roughly one third of the beam FWHM. The mapping was carried out in good weather conditions $(\tau \sim 0.03)$ and a typical system temperature of $T_{\text {sys }} \sim 90 \mathrm{~K}$. The data were processed with the GILDAS software (Pety 2005) and CASA (McMullin et al. 2007).

\section{2. $\mathrm{H}_{2}$}

The Herschel/SPIRE images were extracted from the pipelinereduced images of the Taurus complex made in the course of the Herschel Gould Belt Survey (André et al. 2010). The data were downloaded from the Herschel Science Archive (HSA) ${ }^{1}$. We calculated the $N\left(\mathrm{H}_{2}\right)$ using only the three SPIRE (Griffin et al. 2010) bands at $250 \mu \mathrm{m}, 350 \mu \mathrm{m}$, and $500 \mu \mathrm{m}$, for which the pipeline reduction includes zero-level corrections based on comparison with the Planck satellite data. A modified blackbody function with the dust emissivity spectral index $\beta=1.5$ was fitted to each pixel, after smoothing the $250 \mu \mathrm{m}$ and $350 \mu \mathrm{m}$ images to the resolution of the $500 \mu \mathrm{m}$ image $\left(\sim 40^{\prime \prime}\right)$, and resampling all images to the same grid. For the dust emissivity coefficient we adopted the value from Hildebrand (1983), $\kappa_{250 \mu \mathrm{m}}=$ $0.1 \mathrm{~cm}^{2} \mathrm{~g}^{-1}$. Suutarinen et al. (2013) derived a similar value for $\kappa_{250 \mu \mathrm{m}}$ in the starless core $\mathrm{CrA} \mathrm{C}$. The obtained maximum column density is $N\left(\mathrm{H}_{2}\right)=2.8 \times 10^{22} \mathrm{~cm}^{-2}$, which is comparable to the value of $9.4 \times 10^{22} \mathrm{~cm}^{-2}$ derived in Crapsi et al. (2005) at the $1.2 \mathrm{~mm}$ dust emission peak defined in Ward-Thompson et al. (1999), if we consider the different beams (40" versus 11").

\section{Results and discussion}

\subsection{Chemical differentiation in L1544}

In Fig. 1, the integrated intensities of the $2_{1,2}-1_{1,1}\left(E_{2}\right)$ transition of methanol from Bizzocchi et al. (2014) (red contours) and

\footnotetext{
1 www . cosmos.esa.int/web/herschel/science-archive
}

Table 1. Spectroscopic parameters of the observed lines.

\begin{tabular}{ccccc}
\hline \hline Molecule & $\begin{array}{c}\text { Transition } \\
J_{K_{a}^{\prime}, K_{c}^{\prime}}^{\prime}-J_{K_{a}^{\prime \prime}, K_{c}^{\prime \prime}}^{\prime \prime}\end{array}$ & $\begin{array}{c}\text { Rest frequency } \\
(\mathrm{MHz})\end{array}$ & $\begin{array}{c}E_{u p} \\
(\mathrm{~K})\end{array}$ & $\begin{array}{c}A \\
\left(\times 10^{-5} \mathrm{~s}^{-1}\right)\end{array}$ \\
\hline$c-\mathrm{C}_{3} \mathrm{H}_{2}$ & $3_{2,2}-3_{1,3}$ & 84727.688 & 16.1 & 1.04 \\
$\mathrm{CH}_{3} \mathrm{OH}$ & $2_{1,2}-1_{1,1}\left(E_{2}\right)$ & 96739.362 & $12.53^{a}$ & 0.26 \\
\hline
\end{tabular}

Notes. The map of methanol in L1544 is already presented in Bizzocchi et al. (2014). ${ }^{(a)}$ Energy relative to the ground $0_{0,0}, A$ rotational state.

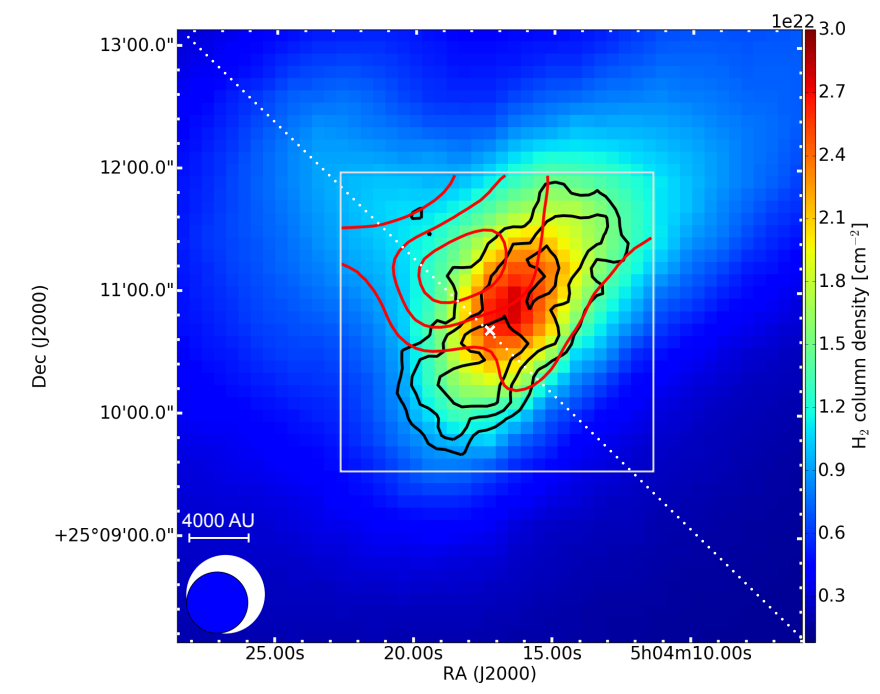

Fig. 1. Integrated intensities of the $2_{1,2}-1_{1,1}\left(E_{2}\right)$ transition of methanol (red contour) and $3_{2,2}-3_{1,3}$ transition of cyclopropenylidene (black contour) are plotted on the $\mathrm{H}_{2}$ column density map derived from farinfrared images observed by Herschel (Pilbratt et al. 2010). The contour levels are $10 \sigma, 15 \sigma$, and $20 \sigma \mathrm{CH}_{3} \mathrm{OH}\left(2 \times 10^{-1}, 3 \times 10^{-1}\right.$ and $4 \times 10^{-1} \mathrm{~K} \mathrm{~km} \mathrm{~s}^{-1}$, respectively) and $5 \sigma, 10 \sigma$, and $15 \sigma c-\mathrm{C}_{3} \mathrm{H}_{2}(4.8 \times$ $10^{-2}, 7.2 \times 10^{-2}$ and $9.6 \times 10^{-2} \mathrm{~K} \mathrm{~km} \mathrm{~s}^{-1}$, respectively). The white box defines the area that was mapped with the $30 \mathrm{~m}$ telescope. The white cross marks the position of the $1.2 \mathrm{~mm}$ dust emission peak from Ward-Thompson et al. (1999). The beams of SPIRE and of the $30 \mathrm{~m}$ telescope are shown as a white and blue circle, respectively. The dotted line represents the positions where the column densities plotted in Fig. 3 have been extracted. Methanol and cyclopropenylidene clearly trace different regions in L1544, and show a chemical differentiation within the prestellar core.

the $3_{2,2}-3_{1,3}$ transition of cyclopropenylidene (black contours) are superimposed on the $\mathrm{H}_{2}$ column density derived from farinfrared images observed by Herschel (Pilbratt et al. 2010). The white box defines the area mapped with the $30 \mathrm{~m}$ telescope.

The $N\left(\mathrm{H}_{2}\right)$ map shows a sharp and straight edge towards the south and south-west part of the cloud, which marks the edge of the filamentary cloud within which L1544 is embedded (see also Tafalla et al. 1998). Therefore, this side of the dense core should be more affected by the photochemistry activated by the interstellar radiation field (ISRF). Methanol shows a complementary distribution with respect to cyclopropenylidene. Is this expected based on our understanding of the chemistry of these two molecules?

Methanol is believed to be formed on dust grains (Watanabe \& Kouchi 2002) by subsequent hydrogenation of carbon monoxide, and its detection towards prestellar cores is already a challenge for current models given the absence of efficient desorption processes in these sources. Thermal desorption is out of question because of the low dust temperature. 


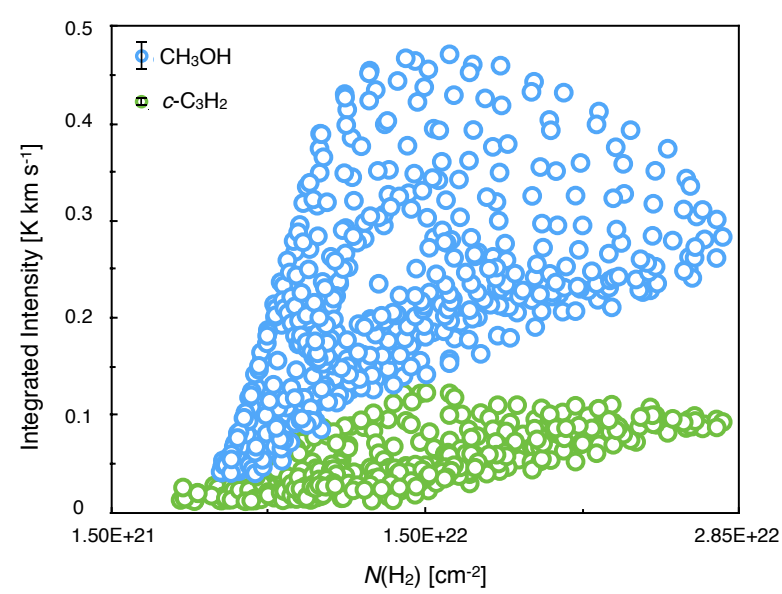

Fig. 2. Scatter plot of the integrated intensities of the $2_{1,2}-1_{1,1}\left(E_{2}\right)$ $\mathrm{CH}_{3} \mathrm{OH}$ line (blue circles) and the $3_{2,2}-3_{1,3} c-\mathrm{C}_{3} \mathrm{H}_{2}$ line (green circles) with respect to the $\mathrm{H}_{2}$ column density inferred from the SPIRE observations (see text). The average error bars are shown in the upper part of the plot. The average errors are $14 \mathrm{mK} \mathrm{km} \mathrm{s}^{-1}$ for $\mathrm{CH}_{3} \mathrm{OH}$ and $4 \mathrm{mK} \mathrm{km} \mathrm{s}{ }^{-1}$ for $c-\mathrm{C}_{3} \mathrm{H}_{2}$. Only the pixels where the integrated intensity over the average error is larger than 3 are plotted.

Recent laboratory studies showed that the photo-desorption of methanol from ices is likewise negligible (Bertin et al. 2016; Cruz-Diaz et al. 2016). The main desorption products when irradiating pure and mixed methanol ices are photo-fragments of methanol. An alternative route to explain the presence of methanol in the gas phase is the reactive/chemical desorption that has been theoretically proposed by Garrod et al. (2007) and Vasyunin \& Herbst (2013) and experimentally studied by Dulieu et al. (2013) and Minissale et al. (2016). On the other hand, $c-\mathrm{C}_{3} \mathrm{H}_{2}$ is mainly formed in the gas phase (e.g. Spezzano et al. 2013) and is expected to preferentially trace dense and chemically young gas, that is, gas where $\mathrm{C}$ atoms have not yet been mainly locked into $\mathrm{CO}$. This gas rich in $\mathrm{C}$ atoms is expected in the outer envelope of an externally illuminated dense core (e.g. Aikawa et al. 2001). However, towards L1544, $c-\mathrm{C}_{3} \mathrm{H}_{2}$ only appears to trace one side of the core, the one closer to the sharp $N\left(\mathrm{H}_{2}\right)$ edge and away from the $\mathrm{CH}_{3} \mathrm{OH}$ peak. This indicates that photo-chemistry is not uniformly active around L1544, most likely because the distribution of the envelope material (belonging to the filament within which L1544 is embedded) is not uniform, as is clearly shown by the Herschel map in Fig. 1. This figure shows that methanol traces a region farther away from the southern sharp edge of the $N\left(\mathrm{H}_{2}\right)$ map, which is possibly more shielded from the ISRF and where most of the carbon is locked in $\mathrm{CO} . \mathrm{CH}_{3} \mathrm{OH}$ is preferentially found at the northern edge of L1544 because here photochemistry does not play a major role (so $\mathrm{C}$ is locked in $\mathrm{CO}$ ) and the density is low enough to maintain a higher fraction of $\mathrm{CH}_{3} \mathrm{OH}$ in the gas phase, but above the threshold value for $\mathrm{CO}$ freeze-out, a few $\times 10^{4} \mathrm{~cm}^{-3}$ (Caselli et al. 1999). Based on the Keto \& Caselli (2010) model (that was updated by Keto et al. 2014), the volume density at the distance of the methanol peak is predicted to be $8 \times 10^{4} \mathrm{~cm}^{-3}$, which is just above the threshold value. In contrast, cyclopropenylidene has the most prominent peak towards the southern sharp edge of the $\mathrm{H}_{2}$ column density and extends along the semi-major axis of the core, almost parallel to the south-west edge of the $N\left(\mathrm{H}_{2}\right)$ map. This behaviour is also clearly shown in Fig. 2, where the integrated intensities of both methanol and cyclopropenylidene are plotted against

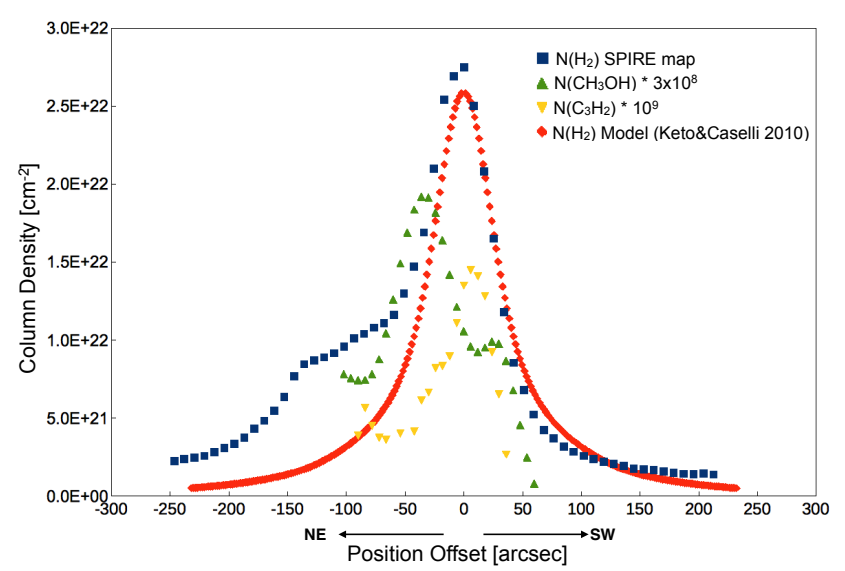

Fig. 3. Column densities of $\mathrm{H}_{2}, c-\mathrm{C}_{3} \mathrm{H}_{2}$ and $\mathrm{CH}_{3} \mathrm{OH}$ extracted along the dotted line present in Fig. 1, as well as the $N\left(\mathrm{H}_{2}\right)$ calculated with the model of L1544 described in Keto \& Caselli (2010) smoothed at 40". While the $N\left(\mathrm{H}_{2}\right)$ presents a sharp drop towards the south-west, its decrease is not as steep towards the north-east. The resulting different illumination on the two sides of L1544 is likely to cause the different distribution of cyclopropenylidene and methanol within the core.

$N\left(\mathrm{H}_{2}\right) . c-\mathrm{C}_{3} \mathrm{H}_{2}$ is also present at values of $N\left(\mathrm{H}_{2}\right)$ that are lower than those of methanol, and it maintains a flat intensity profile, suggestive of a layer-like structure, with no significant increase towards the core centre. The $\mathrm{CH}_{3} \mathrm{OH}$ intensity instead shows a sharp rise up to column densities of about $1.6 \times 10^{22} \mathrm{~cm}^{-2}$, and it declines at higher values.

Figure 3 shows the column densities of $\mathrm{H}_{2}, c-\mathrm{C}_{3} \mathrm{H}_{2}$ and $\mathrm{CH}_{3} \mathrm{OH}$ extracted along the dotted line present in Fig. 1, as well as the $N\left(\mathrm{H}_{2}\right)$ calculated with the model of L1544 described in Keto \& Caselli (2010) assuming a beam size of 40". The column densities of $c-\mathrm{C}_{3} \mathrm{H}_{2}$ and $\mathrm{CH}_{3} \mathrm{OH}$ were calculated assuming that the lines are optically thin, using the formula given in Eq. (1) of Spezzano et al. (2016). We assumed a $T_{\text {ex }}$ of $6 \mathrm{~K}$ for $c-\mathrm{C}_{3} \mathrm{H}_{2}$ and $6.5 \mathrm{~K}$ for $\mathrm{CH}_{3} \mathrm{OH}$, as done in Spezzano et al. (2013) and Bizzocchi et al. (2014), respectively. The same works reported that both lines present moderate optical depths $(\tau<0.4)$. This plot shows that the decrease of $N\left(\mathrm{H}_{2}\right)$ towards the south-west, where cyclopropenylidene is more abundant, is much steeper than towards the north-east, where methanol is more abundant. A full map of the $N\left(c-\mathrm{C}_{3} \mathrm{H}_{2}\right) / N\left(\mathrm{CH}_{3} \mathrm{OH}\right)$ column density ratio can be seen in Fig. A.1, showing a clear peak towards the south-east side of L1544. Figure 3 also shows that $c-\mathrm{C}_{3} \mathrm{H}_{2}$ and $\mathrm{CH}_{3} \mathrm{OH}$ both belong to the same dense core (identified by the brightest peak in $N\left(\mathrm{H}_{2}\right)$ ), but trace different parts of it. We obtain the same result by comparing the line-width and velocity maps of the two molecules, see Fig. B.1. Despite the different spatial distributions, the two molecules trace the same kinematic patterns (velocity gradient and amount of non-thermal motions). This indicates that the velocity fields are dominated by the bulk motions (gravitational contraction and rotation) of the prestellar core, which similarly affect the two sides of the core and do not depend on the chemical composition of the gas. In summary, $c-\mathrm{C}_{3} \mathrm{H}_{2}$ and $\mathrm{CH}_{3} \mathrm{OH}$ trace different parts of the same dense core and no velocity shift is present (unlike for the L1551 case; Swift et al. 2006). We note that the observed transitions of $c-\mathrm{C}_{3} \mathrm{H}_{2}$ and $\mathrm{CH}_{3} \mathrm{OH}$ have relatively high critical densities (between a few $\times 10^{4}$ and $10^{6} \mathrm{~cm}^{-3}$ ), so that these lines are not expected to trace the more diffuse filamentary material surrounding the prestellar core. 


\subsection{Carbon-chain chemistry}

The above results show that carbon-chain chemistry (CCC) is active in the chemically and dynamically evolved prestellar core L1544 in the direction of its maximum exposure to the ISRF (as shown by the sharp edge in the $\mathrm{H}_{2}$ column density map; see Fig. 1). Based on our observations, we conclude that CCC can also be present in slowly contracting clouds such as L1544 (Keto et al. 2015) and that the $\mathrm{C}$ atoms driving the CCC can partially deplete on dust grain surfaces, producing solid $\mathrm{CH}_{4}$, which is the origin of the so-called warm carbon-chain chemistry (WCCC; Sakai \& Yamamoto 2013), when it is evaporated in the proximity of newly born young stellar objects. This apparently contradicts the suggestion that WCCC becomes active when the parent core experiences a fast contraction on a timescale close to that of free fall (Sakai et al. 2008, 2009). In these previous papers, fast contraction was invoked to allow $\mathrm{C}$ atoms to deplete onto dust grains before they are converted to $\mathrm{CO}$ in the gas phase (Sakai \&Yamamoto 2013), so that a substantial amount of solid $\mathrm{CH}_{4}$ is produced.

Based on the results of our observations, we propose an alternative view to the WCCC origin that is not depending on the dynamical evolution: cores embedded in low-density (and low- $A_{v}$ ) environments, where the ISRF maintains a large portion of the carbon in atomic form in most of the surrounding envelope, become rich in solid $\mathrm{CH}_{4}$ and carbon chains, precursors to WCCC. Cores such as L1544, which are embedded in non-uniform clouds, with non-uniform amounts of illumination from the ISRF, are expected to have mixed ices, where $\mathrm{CH}_{4}$ and $\mathrm{CH}_{3} \mathrm{OH}$ ices coexist. It is interesting to note that $\mathrm{CH}_{3} \mathrm{OH}$ has recently been found to correlate with the $\mathrm{C}$-chain molecule $\mathrm{C}_{4} \mathrm{H}$ towards embedded protostars (Graninger et al. 2016), suggesting that these two classes of molecules can indeed coexist. We suggest that the sources studied by Graninger et al. (2016) had a non-uniform environment similar to those around L1544, whereas the WCCC sources formed within prestellar cores with most of their envelope affected by photochemistry (due to a lower overall $\mathrm{H}_{2}$ column density or extinction towards their outer edges). We are now extending our study of $\mathrm{CH}_{3} \mathrm{OH}$ and $c-\mathrm{C}_{3} \mathrm{H}_{2}$ to a larger sample of starless cores and link the results to Herschel $N\left(\mathrm{H}_{2}\right)$ maps, to determine whether the conclusion reached for L1544 can be extended to other regions.

\section{Summary}

We have compared the spatial distribution of $c-\mathrm{C}_{3} \mathrm{H}_{2}$ and methanol and underlined a chemical differentiation within L1544. While cyclopropenylidene peaks towards the sharp edge of the $\mathrm{H}_{2}$ column density map, where a fraction of the carbon is still in atomic form, methanol has its maximum away from this edge. This suggests that active carbon chemistry driven by free carbon is at work on the south-western side of L1544, which is exposed to the ISRF. Because of the high density, accretion of carbon atoms onto grains probably is effective in this region, and ices may become rich in $\mathrm{CH}_{4}$. On the opposite side, carbon is mostly locked in $\mathrm{CO}$ and methanol is formed on the surface of dust grains following $\mathrm{CO}$ freeze-out. Here, ices are expected to be rich in $\mathrm{CO}$ and methanol. The two sides of the core follow the same dynamics, however, and it is expected that mixed ices will be present towards the core centre, where a protostar will form. This mixing would provide a natural explanation for the correlation between $\mathrm{CH}_{3} \mathrm{OH}$ and $\mathrm{C}_{4} \mathrm{H}$ found recently in a sample of embedded protostars.

Acknowledgements. The authors wish to thank the anonymous referee for useful suggestions and Ana Chacón-Tanarro for useful discussions. P.C. acknowledges the financial support of the European Research Council (ERC; project PALs 320620). S.B. acknowledges financial support from the German Science Foundation (DFG) in the framework of the priority program SPP 1573 (grant BR 4287/1-2).

\section{References}

Aikawa, Y., Ohashi, N., Inutsuka, S.-I., Herbst, E., \& Takakuwa, S. 2001, ApJ, 552,639

André, P., Men'shchikov, A., Bontemps, S., et al. 2010, A\&A, 518, L102

Bertin, M., Romanzin, C., Doronin, M., et al. 2016, ApJ, 817, L12

Bizzocchi, L., Caselli, P., Spezzano, S., \& Leonardo, E. 2014, A\&A, 569, A27

Caselli, P., Walmsley, C. M., Tafalla, M., Dore, L., \& Myers, P. C. 1999, ApJ, 523, L165

Caselli, P., Walmsley, C. M., Zucconi, A., et al. 2002, ApJ, 565, 331

Ciolek, G. E., \& Basu, S. 2000, ApJ, 529, 925

Crapsi, A., Caselli, P., Walmsley, C. M., et al. 2005, ApJ, 619, 379

Crapsi, A., Caselli, P., Walmsley, M. C., \& Tafalla, M. 2007, A\&A, 470, 221

Cruz-Diaz, G. A., Martín-Doménech, R., Muñoz Caro, G. M., \& Chen, Y.-J. 2016, A\&A, 592, A68

Dulieu, F., Congiu, E., Noble, J., et al. 2013, Scientific Reports, 3, 1338

Garrod, R. T., Wakelam, V., \& Herbst, E. 2007, A\&A, 467, 1103

Graninger, D. M., Wilkins, O. H., \& Öberg, K. I. 2016, ApJ, 819, 140

Griffin, M. J., Abergel, A., Abreu, A., et al. 2010, A\&A, 518, L3

Hildebrand, R. H. 1983, Quant. J. Roy. Astron. Soc., 24, 267

Keto, E., \& Caselli, P. 2010, MNRAS, 402, 1625

Keto, E., Caselli, P., \& Rawlings, J. 2015, MNRAS, 446, 3731

Larson, R. B. 1969, MNRAS, 145, 271

McMullin, J. P., Waters, B., Schiebel, D., Young, W., \& Golap, K. 2007, Astronomical Data Analysis Software and Systems XVI, 376, 127

Minissale, M., Dulieu, F., Cazaux, S., \& Hocuk, S. 2016, A\&A, 585, A24

Ohashi, N., Lee, S. W., Wilner, D. J., \& Hayashi, M. 1999, ApJ, 518, L41 Penston, M. V. 1969, MNRAS, 144, 425

Pety, J. 2005, in SF2A-2005: Semaine de l'Astrophysique Française, 721 (EDP Sciences)

Pilbratt, G. L., Riedinger, J. R., Passvogel, T., et al. 2010, A\&A, 518, L1

Sakai, N., \& Yamamoto, S. 2013, Chem. Rev., 113, 8981

Sakai, N., Sakai, T., Hirota, T., \& Yamamoto, S. 2008, ApJ, 672, 371

Sakai, N., Sakai, T., Hirota, T., Burton, M., \& Yamamoto, S. 2009, ApJ, 697, 769

Shu, F. H. 1977, ApJ, 214, 488

Spezzano, S., Brünken, S., Schilke, P., et al. 2013, ApJ, 769, L19

Spezzano, S., Gupta, H., Brünken, S., et al. 2016, A\&A, 586, A110

Suutarinen, A., Haikala, L. K., Harju, J., et al. 2013, A\&A, 555, A140

Suzuki, H., Yamamoto, S., Ohishi, M., et al. 1992, ApJ, 392, 551

Swift, J. J., Welch, W. J., Di Francesco, J., \& Stojimirović, I. 2006, ApJ, 637, 392

Tafalla, M., Mardones, D., Myers, P. C., et al. 1998, ApJ, 504, 900

Tafalla, M., Myers, P. C., Caselli, P., Walmsley, C. M., \& Comito, C. 2002, ApJ, 569,815

Tafalla, M., Myers, P. C., Caselli, P., \& Walmsley, C. M. 2004, A\&A, 416, 191

Tafalla, M., Santiago-García, J., Myers, P. C., et al. 2006, A\&A, 455, 577

Vasyunin, A. I., \& Herbst, E. 2013, ApJ, 769, 34

Ward-Thompson, D., Motte, F., \& Andre, P. 1999, MNRAS, 305, 143

Watanabe, N., \& Kouchi, A. 2002, ApJ, 571, L173 


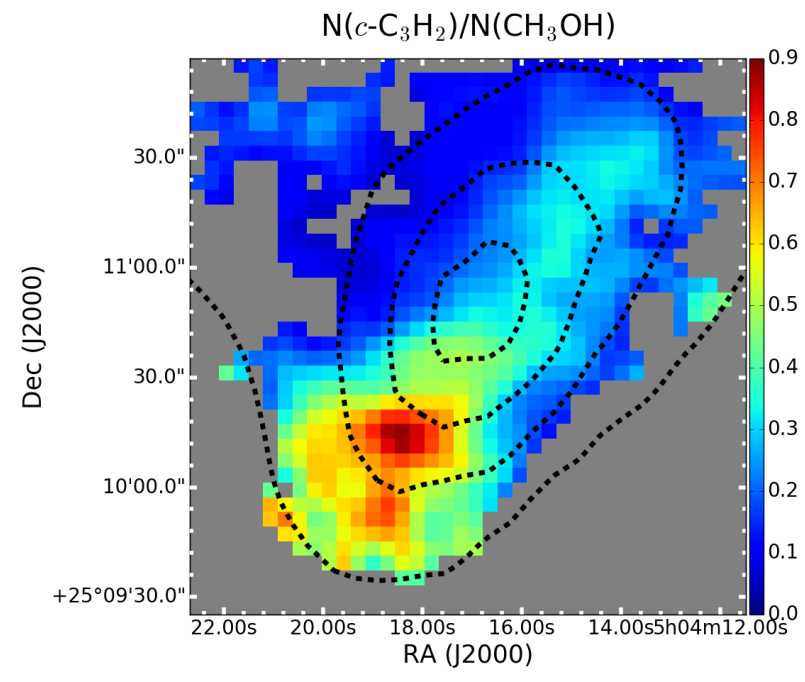

Fig. A.1. Maps of the total column density ratio of cyclopropenylidene and methanol. The black dashed line represent the $90 \%, 70 \%, 50 \%$, and $30 \%$ of the $\mathrm{H}_{2}$ column density peak value derived from Herschel maps.

\section{Appendix A: Map of the $N\left(c-\mathrm{C}_{3} \mathrm{H}_{2}\right) / N\left(\mathrm{CH}_{3} \mathrm{OH}\right)$ ratio}

Figure A. 1 shows a map of the column density ratio of $c-\mathrm{C}_{3} \mathrm{H}_{2}$ and $\mathrm{CH}_{3} \mathrm{OH}$. Only the pixels where the integrated intensity over the average error is larger than 3 are plotted. While both molecules are depleted towards the centre, their abundance ratio is not constant across the core because they present a different distribution. The peak value of the $N\left(c-\mathrm{C}_{3} \mathrm{H}_{2}\right) / N\left(\mathrm{CH}_{3} \mathrm{OH}\right)$ ratio is towards the south-east edge of the cloud.

\section{Appendix B: Kinematics}

The frequency resolution is high enough to reveal some interesting features on the core kinematics from the line profiles. The upper panels in Fig. B.1 show the line-width maps of $c-\mathrm{C}_{3} \mathrm{H}_{2}$ and $\mathrm{CH}_{3} \mathrm{OH}$, while the lower panels show the $V_{\mathrm{LSR}}$ maps. The black dashed lines represent the contours of the $\mathrm{H}_{2}$ column density. Here the line width corresponds to the intensity-weighted dispersion of the velocity $(\sigma)$. The line width of both molecules is larger along the major axis of L1544 (south-east to north-west).
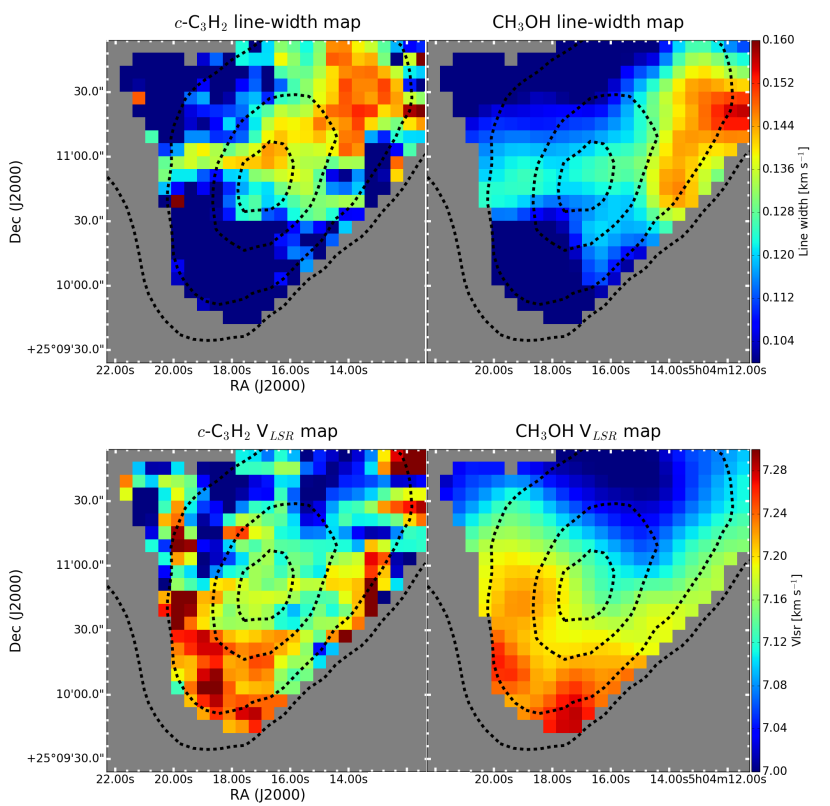

Fig. B.1. Maps of the line width (upper panels) and $V_{\mathrm{LSR}}$ (lower panels) of cyclopropenylidene and methanol. The velocity and line-width structure is similar in the two tracers (see text).

In addition to the well-known line-width increase measured in $\mathrm{N}_{2} \mathrm{H}^{+}(1-0)$ lines towards the centre of the core that is due to contraction motions (Caselli et al. 2002), cyclopropenylidene and methanol also show some line broadening towards the northwest and towards the east. This may suggest the presence of a non-uniform turbulent field, possibly caused by non-uniform accretion of envelope material onto the core. The $V_{\text {LSR }}$ maps clearly show a gradient from the north to the south of the core, with the velocity increasing towards the south part of L1544. The line profiles predicted by the model from Ciolek \& Basu (2000) shown in Fig. 6 in Caselli et al. (2002) show the same behaviour, and to some extent also the observed line profiles, of $\mathrm{N}_{2} \mathrm{H}^{+}$in Fig. 7 of the same paper. Therefore, despite the different distributions of $c-\mathrm{C}_{3} \mathrm{H}_{2}$ and $\mathrm{CH}_{3} \mathrm{OH}$ in $\mathrm{L} 1544$, the two molecules trace similar kinematic patterns. 